\title{
Correction to: Blood lymphocyte subsets identify optimal responders to IFN-beta in MS
}

\author{
Raquel Alenda ${ }^{1,6} \cdot$ Lucienne Costa-Frossard $^{2,6} \cdot$ Roberto Alvarez-Lafuente $^{3,6}$. Carmen Espejo ${ }^{4,5,6}$. \\ Eulalia Rodríguez-Martín ${ }^{1,6}$. Susana Sainz de la Maza ${ }^{2,6}$. Noelia Villarrubia ${ }^{1,6}$. Jordi Río ${ }^{4,5,6}$. \\ María I. Domínguez-Mozo ${ }^{3,6} \cdot$ Xavier Montalban ${ }^{4,5,6}$. José C. Álvarez-Cermeño ${ }^{2,6} \cdot$ Luisa M. Villar ${ }^{1,6}$ (1)
}

Published online: 24 November 2017

(c) Springer-Verlag GmbH Germany, part of Springer Nature 2017

\section{Correction to: J Neurol}

https://doi.org/10.1007/s00415-017-8625-6

The author claims that his name is incorrectly listed on PubMed. It seems that the first and last name has been mixed up.

Correct first name is: Susana (on PubMed: S.S.).

Correct last name is: Sainz de la Maza (on PubMed: de la Maza).

On SpringerLink the name is listed correctly, but on PubMed he is listed as de la Maza S.S.

The original article can be found online at https://doi. org/10.1007/s00415-017-8625-6.

Luisa M. Villar

luisamaria.villar@salud.madrid.org

1 Servicio de Inmunología, Hospital Universitario Ramón y Cajal, IRYCIS, Ctra. Colmenar km 9.100, 28034 Madrid, Spain

2 Servicio de Neurología, Hospital Universitario Ramón y Cajal, IRYCIS, Madrid, Spain

3 Servicio de Neurología, Hospital Clínico San Carlos, IdISSC, Madrid, Spain

4 Servei de Neurologia-Neuroimmunologia, Centre d'Esclerosi Múltiple de Catalunya, Vall d'Hebron Institut de Recerca, Hospital Universitari Vall d'Hebron, Barcelona, Spain

5 Universitat Autònoma de Barcelona, Barcelona, Spain

6 Red Española de Esclerosis Múltiple (REEM), Fondo de Investigación Sanitaria, Instituto de Salud Carlos III, Ministerios de Economía y Competitividad, Madrid, Spain 